\title{
B-spline sectional model for general 3D effects in reinforced concrete elements
}

\author{
Mauro Poliotti, Jesús-Miguel Bairán* \\ Department of Civil and Environmental Engineering, Universitat Politècnica de \\ Catalunya, (UPC), Jordi Girona, 1-3, Campus Nord, Mod. C-1, 08034 Barcelona, Spain
}

\begin{abstract}
In this paper, an efficient sectional model for the nonlinear analysis of reinforced concrete elements sensible to 3D stress-components effects is presented. The classic plane-sections kinematic hypothesis is enhanced with a warping-distortion displacement field, which enables the model to reproduce the interaction between normal and tangential forces. The complementary field is obtained explicitly considering the inter-fiber equilibrium. This is solved using b-splines interpolation on the cross-section domain. The proposed method significantly reduces the number of unknowns compared with a finite element solution. The model is able to reproduce the interaction of longitudinal and transverse reinforcement with the concrete matrix. The validation shows that the presented model reproduces accurately complex failure modes as pure shear and coupling between bending and torsion. Further, as the transverse reinforcement is considered explicitly, confinement can be simulated in an objective manner. The presented model is an efficient tool for nonlinear analysis of reinforced concrete sections under general loading.
\end{abstract}

Keywords: Nonlinear sectional analysis, Reinforced concrete, B-splines, Interaction, Shear, Torsion, Confinement.

\footnotetext{
${ }^{*}$ Corresponding author

Email addresses: mauro.poliotti@upc.edu (Mauro Poliotti), jesus.miguel.bairan@upc.edu (Jesús-Miguel Bairán )

1

Please cite this paper as follows:

Poliotti M, Bairan JM, "B-spline sectional model for general 3D effects in reinforced concrete elements", Engineering Structures, 2020, doi.org/10.1016/j.engstruct.2020.110200
} 


\section{Introduction}

Structural elements where one dimension is much larger than the others can be assimilated as linear elements, this is the case of most slender beams and columns. The overall element behaviour can be obtained by integration of the sectional response on each cross section along the elements axis [1-3]. This type of elements allows a simple representation of complex structural systems and lead to efficient pre- and post-processing compared with the alternative solid three-dimensional models. Further, 1D elements are closer to the engineering practice, as most codes and provisions [4-6] are orientated to the design or assessment of one dimensional elements as beams or columns, and its cross section domain. Guidelines on the design of structural elements under 3D states are scant.

The accuracy of 1D elements depends on the proper representation of the behaviour of each cross section. Traditionally, the plane-section kinematic hypothesis of Navier-Bernoulli is made in conjunction with uniaxial fiber discretization of the cross-section $[3 ; 7 ; 8]$. This enables the model to efficiently reproduce the interaction between bending moments and the axial load. Robust beam models were developed following this approach $[2 ; 3 ; 7-$ 9], and were applied to the analysis of reinforced concrete structures where the failure was principally due to axial loads or bending moments.

The main shortcoming of this type of models is that the interaction between normal (axial load and bending moments) and tangential (shear loads and torsion) forces is neglected [10-12]. This causes a loss of accuracy in elements with high shear forces or torsional moment, especially if anisotropic materials are involved. It has also been shown in $[13 ; 14]$ that anisotropy leads to an explicit dependency of axial stresses on the shear force. In particular, in this paper the applications are focused on the problem of cracked-induced anisotropy of concrete, which controls the interaction between normal and shear stresses, as described in $[12 ; 15-17]$.

Moreover, classic fiber-beam models only consider longitudinal fibers. In typical reinforced concrete sections they represent the concrete mass and longitudinal reinforcements, and do not consider transverse elements, as stirrups or jackets. These transverse elements provide different shear strength mechanisms. Further, they constrain the concrete transverse expansion in compressive cases, providing a confining pressure, which increases both the strength and ductility of concrete. In classic-fiber-beam models $[3 ; 7 ; 8]$, the transverse reinforcements is accounted for at the material level by means of 
reinforcement ratios in empirically based $1 \mathrm{D}$ constitutive laws.

The need of reproducing different failure modes, as shear or torsional failures, as well as capturing the role of transverse reinforcements, became of special interest the last years with the assessment and reinforcement of existing structures and with the increasing demand of nonlinear analysis by the new codes and provisions [11]. This motivated several researchers to study the coupling between normal and tangential forces and to develop models that take into account different degrees of interaction, $[11 ; 18-26]$.

In this paper, the sectional model proposed by [20-22] is modified using a novel numerical technique, which substantially improves the computational performance and requires less number of internal degrees of freedom for a similar accuracy. Here, a complementary displacement field which enables the model to reproduce the total interaction, is obtained as a weighted sum of b-splines functions defined in the cross-section domain. In this way, the number of degrees of freedom involved is significantly reduced in comparison with a finite element $(F E)$ solution. The presented model is then validated through a series of tests cases where the capabilities to reproduce tangential forces are shown. Further, the model is applied to study confinement in reinforced concrete sections where the interaction between concrete and transverse reinforcements is essential. Finally, main conclusions are drawn.

\section{Multiaxial interaction in frame elements}

The robustness of fiber-beam elements, and the increasing need of reproducing complex failure modes that involve the coupling between normal and tangential internal forces, have motivated researchers to extend the classical fiber-beam models to include different degrees of interaction between internal forces. Extended state of the art reviews can be found in Ceresa et al. [11] and Bairán and Marí [12]. Here, only models based on inter-fiber equilibrium are briefly reviewed as they showed to be able to accurately reproduce different failure modes.

Vecchio and Collins [18] developed a dual-section analysis for the case of $2 \mathrm{D}$ frame elements. The coupling between longitudinal and transverse stresses is obtained by explicitly considering the equilibrium between fibers, which involves the analysis of two adjacent cross-sections to approximate the longitudinal stress gradient. The dual-section model is a non local model, as it needs information from adjacent points, so an ad-hoc element formulation is needed. To overcome this problem, Bentz [19] introduced the Longitudinal 
Stiffness Method, where the longitudinal stress gradient was calculated locally considering equilibrium equations at the beam level. This method is a local sectional model, but only for $2 \mathrm{D}$ elements.

Later, Bairán and Marí [20, 21, 22] presented a sectional model called TINSA (Total Interaction Nonlinear Sectional Analysis). It is based on the enhancement of the kinematic field of the Navier-Bernoulli theory by means of a warping-distortion displacement field which is obtained by solving the inter-fiber equilibrium in the section domain with a $2 \mathrm{D}$ FE model. The warping-distortion field considered is independent of the $x$-coordinate neglecting its variation along the beam length, this leads to a sectional model that is completely independent of the frame formulation, thus there is no need for additional degrees of freedom at the beam level. A consequence of this assumption is that the effect of non-uniform warping and shear lag effects are neglected, but this is relevant in thin-walled sections rather than in compact cross sections as is the case of most reinforced concrete beams and columns. This formulation proved to capture the interaction between the six possible internal forces in sections of any shape and takes into account both longitudinal and transverse reinforcements explicitly. Its main drawback is the higher computational demand compared with traditional beam models.

Mohr et al. [23], presented a modification of the TINSA model for the case of 2D frames. The complementary displacement field is calculated as a weighted sum of Taylor's polynomials defined in the section height. This method avoids the FE solution, reducing the computational cost of the original model. The sectional model was implemented on a flexibility-based frame element and tested in beams with bending moment and shear. As it was developed for 2D frames, no torsion or bidirectional shear flows can be analysed with this model.

Le Corvec [24] and Di Re [25] presented 3D frame elements based also on the displacement decomposition. As an additional hypothesis to the original formulation, they presented a complementary displacement field, which only produces warping neglecting the distortion of the cross-section. The out of plane displacement is obtained by interpolation over the section domain but also on the beam length, this allows the model to capture non uniform warping and shear lag effects. As interpolation function over the cross section domain, Le Corvec [24] used Lagrange's polynomials, while Di Re [25] included Hermite's polynomials. Both models were implemented in 3D force-based elements based on the $\mathrm{Hu}$-Washizu variational principle. These models can capture the interaction of the internal forces in a tri-dimensional 
element, but the hypothesis of considering a complementary field with only warping has the drawback that distortion of the section is not captured, so transverse reinforcement is not considered explicitly. Besides, as the complementary field is interpolated on the beam length, a special frame formulation is developed with more degrees of freedom at the beam level that have to be considered or properly condensed.

Kagermanov and Ceresa [26], presented a 3D frame element based on the Timoshenko beam theory that accounts for warping effects with the SaintVenant theory of torsion. There, the warping sectional function was obtained by means of solving the boundary value problem with a FE sectional model. The model is able to capture interaction between the internal forces, but it relies on a fixed strain pattern so it neglects inter-fiber equilibrium.

Based on the previous analysis, the following aspects on the enhanced 1D elements for non-linear concrete analysis are still open; their robustness and computational demand, their ability to trace different failure modes, such as shear and confinement failures, in an objective manner for different geometries and reinforcement arrangements. This motivates the development of a new sectional model, which is the main objective of this research.

\section{Proposed model formulation}

\subsection{Basic features}

In the following, the sectional model proposed by [20-22] is modified by the introduction of a novel numerical technique in order to reduce its computational demand. The problem is focused on a frame element where the $x$ coordinate coincides with the frame axis and the cross-section is defined on the $y$ - $z$ plane. The proposed model neglects the boundary effects, so the hypothesis that the domain is far enough of the discontinuity regions is made. The main hypothesis is the decomposition of the displacement field into two parts: a displacement field that follows the plane-sections kinematic hypothesis of the Navier-Bernoulli theory $\left(\boldsymbol{u}_{p s}\right)$, and a complementary field that captures the sections distortion and warping $\left(\boldsymbol{u}_{w}\right)$. Thus, the total displacement field is obtained as follows:

$$
\boldsymbol{u}=\boldsymbol{u}_{p s}+\boldsymbol{u}_{w}=\left\{\begin{array}{c}
u_{p s} \\
v_{p s} \\
w_{p s}
\end{array}\right\}+\left\{\begin{array}{c}
u_{w} \\
v_{w} \\
w_{w}
\end{array}\right\}
$$


This decomposition can be done straightforwardly in strains, as small strains are assumed, see Eq.(2). Regarding stresses, in the case of nonlinear materials, the same decomposition is valid in an incremental format, and after the integration along the load steps the decomposition can be done without losing generality as in Eq.(3).

$$
\begin{gathered}
\boldsymbol{\varepsilon}=\boldsymbol{\varepsilon}_{p s}+\boldsymbol{\varepsilon}_{w} \\
\boldsymbol{\sigma}=\boldsymbol{\sigma}_{p s}+\boldsymbol{\sigma}_{w}
\end{gathered}
$$

As it was previously stated, to obtain the complementary field, the interfiber equilibrium is considered explicitly. To do so, first the 3D equilibrium equation of a beam is posed in its weak form, as in Eq.(4). Then, the equilibrium residual of a differential element $R(x)$ can be identified, see Eq.(5)

$$
\iiint_{\Omega} \operatorname{div}(\boldsymbol{\sigma}) \delta \boldsymbol{u} d \Omega
$$

intended

$$
\int_{0}^{L}\left(\iint_{A} \operatorname{div}(\boldsymbol{\sigma}) \delta \boldsymbol{u} d A\right) d x=\int_{0}^{L} R(x) d x
$$

The projection of the residual into both displacements fields allows the identification of two equilibrium levels: the beam level represented by the projection of the residual in the plane-section displacement field $R_{p s}$, and the sectional level which implies the projection of the residual in the complementary field $R_{w}$.

$$
\begin{aligned}
& R_{p s}(x)=\iint_{A} \operatorname{div}(\boldsymbol{\sigma}) \delta \boldsymbol{u}_{p s} d A=0 \\
& R_{w}(x)=\iint_{A} \operatorname{div}(\boldsymbol{\sigma}) \delta \boldsymbol{u}_{w} d A=0
\end{aligned}
$$

In order to solve the system in Eqs.(6-7), first $R_{w}=0$ is posed at each cross-section of the beam, and then the beam equilibrium $R_{p s}=0$ is solved. Solving the equilibrium in this way, leads to the full 3D stress tensor, taking into account explicitly the inter-fiber equilibrium.

The main unknown of the sectional model is the warping-distortion displacement field $\left(\boldsymbol{u}_{w}\right)$. To assure the uniqueness of the solution, it is established that the two types of displacement fields should be orthogonal to each other [20]. In order to approximate the complementary field, an additional 
hypothesis is made; namely, the warping-distortion field is independent of the $x$-coordinate neglecting its variation along the beam length, see Eq.(8).

$$
\boldsymbol{u}_{w}=\boldsymbol{u}_{w}(y, z)=\left\{\begin{array}{c}
u_{w}(y, z) \\
v_{w}(y, z) \\
w_{w}(y, z)
\end{array}\right\}
$$

This enables the definition of a cross-sectional model that can be used as a response model of any standard 1D frame formulation. The model solves the sectional equilibrium, Eq.(7), and relates the generalized beam strains with the internal forces, or generalized stresses, see Eq.(9).

$$
\boldsymbol{\epsilon}_{s}=\left\{\begin{array}{c}
\varepsilon_{0} \\
\gamma_{y} \\
\gamma_{z} \\
\phi_{x} \\
\phi_{y} \\
\phi_{z}
\end{array}\right\} \Leftrightarrow \boldsymbol{s}_{s}=\left\{\begin{array}{c}
N \\
V_{y} \\
V_{z} \\
T_{x} \\
M_{y} \\
M_{z}
\end{array}\right\}
$$

Once the sectional equilibrium is achieved, the full stress tensor is obtained on each material point of the cross-section. To obtain the internal forces $\boldsymbol{s}_{s}$, the integration of stresses on the section domain is made considering the virtual work principle. This definition assures that the obtained generalized stresses are energetically conjugated to the generalized strains, see Eq.(10).

$$
\boldsymbol{s}_{s}=\iint_{A} \boldsymbol{B}_{p s}^{T} \boldsymbol{\sigma} d A+\boldsymbol{\Omega}^{T} \boldsymbol{\Xi}^{T} \boldsymbol{A}^{* T} \iint_{A} \boldsymbol{B}_{w}^{T} \boldsymbol{\sigma} d A
$$

Where $\boldsymbol{B}_{p s}$ and $\boldsymbol{B}_{w}$ are strain interpolation matrix that relate the generalized beam strains with the strains on each material point of the section, see Eq.(11). $\boldsymbol{A}^{*}, \boldsymbol{\Xi}$ and $\boldsymbol{\Omega}$ are transformation matrices that stands for internal equilibrium conditions, condensation of redundant degrees of freedom and definition of generalized shear strains, respectively. A detailed derivation of the matrices for the general case of non-isotropic materials can be found in [20; 22].

$$
\begin{aligned}
\varepsilon_{p s} & =\boldsymbol{B}_{p s} \boldsymbol{\epsilon}_{s} \\
\boldsymbol{\varepsilon}_{w} & =\boldsymbol{B}_{w} \boldsymbol{\epsilon}_{s}
\end{aligned}
$$


The use of the sectional model as a response model on any point of a beam needs the definition of a sectional stiffness matrix $\boldsymbol{K}_{s}$, which is computed as in Eq.(12). Where $\boldsymbol{D}$ is a general material constitutive matrix.

$$
\begin{aligned}
\boldsymbol{K}_{s} & =\iint_{A} \boldsymbol{B}_{p s}^{T} \boldsymbol{D} \boldsymbol{B}_{p s} d A+\left(\iint_{A} \boldsymbol{B}_{p s}^{T} \boldsymbol{D} \boldsymbol{B}_{w} d A\right) \boldsymbol{A} \boldsymbol{\Xi} \boldsymbol{\Omega} \\
& +\boldsymbol{\Omega}^{T} \boldsymbol{\Xi}^{T} \boldsymbol{A}^{* T}\left(\iint_{A} \boldsymbol{B}_{w}^{T} \boldsymbol{D} \boldsymbol{B}_{p s} d A\right) \\
& +\boldsymbol{\Omega}^{T} \boldsymbol{\Xi}^{T} \boldsymbol{A}^{* T}\left(\iint_{A} \boldsymbol{B}_{w}^{T} \boldsymbol{D} \boldsymbol{B}_{w} d A\right) \boldsymbol{A} \boldsymbol{\Xi} \boldsymbol{\Omega}
\end{aligned}
$$

\subsection{Numerical solution}

Solving the sectional problem needs a proper space discretization. The original model proposed by [20], solved the problem using a standard 2D FE model, where bidimensional elements represented the solid matrix, linear elements represented transverse reinforcements and point element represented longitudinal reinforcements. The FE solution involves a high number of degrees of freedom (DOF) to solve the sectional problem in most applications, increasing the computational demand. A different approach is proposed here to reduce the DOF involved in the solution.

The warping-distortion complementary field in Eq.(8) is approximated by a weighted sum of predefined functions, see Eq.(13). This reduces significantly the number of degrees of freedom involved to solve the sectional problem compared with the FE solution.

$$
\boldsymbol{u}_{w}=\left\{\begin{array}{c}
u_{w} \\
v_{w} \\
w_{w}
\end{array}\right\}=\left\{\begin{array}{c}
\sum a_{i} F_{i}(y, z) \\
\sum b_{i} F_{i}(y, z) \\
\sum c_{i} F_{i}(y, z)
\end{array}\right\}
$$

The weight factors $\left(a_{i}, b_{i}, c_{i}\right)$ represent the unknowns of the problem. The most classical interpolation functions relies on Lagrange's functions. For a given interpolation grid, the interpolation is performed first independently on each geometric coordinate, then the surface in the section domain is obtained by the tensor product. This method produces one function for each point of the interpolation grid. However, when the number of points of the interpolation grid increases, the order of the Lagrange polynomial increases as well. This may lead to high order polynomials in practical cases, introducing undesired oscillations augmenting the interpolation error locally. 
In order to overcome this issue, piecewise polynomials are used which allows controlling the order of the polynomial in spite the number of grid points. A family of these polynomials are known as splines, they account for continuity conditions in the boundary of the intervals defined by the interpolation grid, and has proven to be efficient and robust [27]. Thanks to that, their use in FE element frameworks has increased in the last years [28-30].

B-splines (basis splines) are constructed from a knot vector which contains their coordinates in increasing order, $\boldsymbol{Z}=\left\{\zeta_{1}, \zeta_{2}, \ldots, \zeta_{n+p+1}\right\}$, where $p$ is the polynomial order and $n+1$ is the number of basis functions. In Eq.(14) the recursive form proposed by [31] is formulated for the $i$-th basis function.

$$
\begin{array}{r}
N_{i, p}(\zeta)=\frac{\zeta-\zeta_{i}}{\zeta_{i+p}-\zeta_{i}} N_{i, p-1}(\zeta)+\frac{\zeta_{i+p+1}-\zeta}{\zeta_{i+p+1}-\zeta_{i+1}} N_{i+1, p-1}(\zeta) \\
N_{i, 0}=\left\{\begin{array}{l}
1 \text { if } \zeta_{i} \leq \zeta<\zeta_{i+1} \\
0 \text { otherwise }
\end{array}\right.
\end{array}
$$

If the value of a function in the boundaries of the knot vector is to be interpolated, an open knot vector with multiplicity of the first and last value is needed. In this work, cubic polynomials are used. Then, for an interpolation grid of $n$ non-repeated elements $n+2$ basis functions can be constructed on each coordinate. Further, the interpolation surface is obtained by the tensor product, see Eq.(15).

$$
F_{i j, p q}(y, z)=\boldsymbol{P}_{i j} N_{i, p}(y) M_{j, q}(z)
$$

For a given cross-section geometry, a number of points has to be selected in order to build the interpolation grid. The b-spline functions are constructed using Eq.(14). The set of b-spline functions constitutes the shape functions defined over the cross section. Fig.(1) shows the grid point selected for a square section and one of the basis functions used in the interpolation. This process is done for each component of the complementary displacement field.

The midpoint integration rule is used to solve the sectional problem as in classical fiber-beam models. Each integration point represents a material point or a fiber. The solid part of the cross section is represented by fibers with 3D constitutive laws and are defined by their geometrical coordinates $(y, z)$ and their projected area on the section. The transverse and longitudinal reinforcements are represented by fibers with uniaxial constitutive 


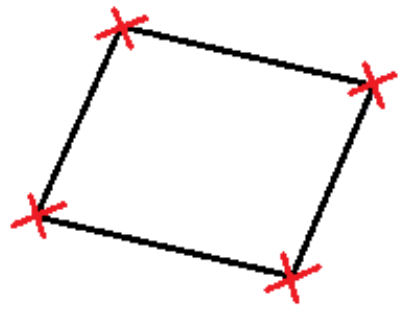

(a)

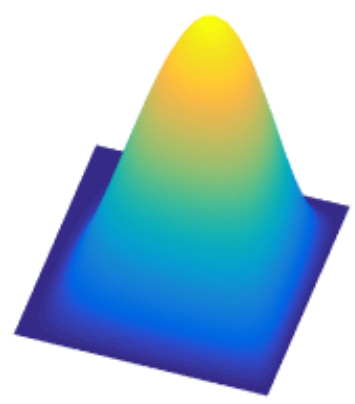

(b)

Figure 1: Bspline Interpolation a) Interpolation grid, b) Basis function

laws. Furthermore, fibers are defined by their position, their projected area in the cross-section plane, and by their inclination with respect to the beam axis. This allows the model to include inclined transverse or longitudinal bars indistinctly. The sectional model algorithm can be seen in Appendix A.

\section{Validation}

In order to verify the accuracy and test the capabilities of the proposed model, two validation sets are presented in the following. The first validation set involves three sections under tangential forces, namely: a triangular elastic section in pure torsion, a reinforced concrete section tested in pure shear and a reinforced concrete box section under combined bending and torsion moments. These three cases are numerically simulated with the proposed model. The FE solution with the TINSA model [20] is also presented. In the case of the elastic triangle in torsion, the obtained response is compared against a theoretical result. In both reinforced concrete sections, experimental results available in literature are contrasted with the numerical simulations.

The second set of validation comprises three sections under axial loading with different confining materials and transverse reinforcement arrangements. First, a reinforced concrete circular section winch is transversally reinforced by a steel spiral is tested. Further, a circular section wrapped with a CFRP jacket is simulated. Finally, a rectangular concrete filled tube is analyzed. 
The results obtained with the proposed model are compared against experimental data available in literature.

In the five cases concerning reinforced concrete sections, perfect bond is assumed. The constitutive law for the steel fibers is a 1D elastic-plastic model with kinematic hardening. Concerning the constitutive modelling of concrete, it is worth mentioning that the presented sectional model can be used with any tri-dimensional constitutive law. If different failures modes are to be reproduced, the constitutive model has to be able to trace a wide range of loading conditions namely shear stresses and high confinement. To this end, the plastic-damage model with evolutive dilatancy introduced in [32] is used in this work. The evolutive dilatancy parameter presented there, proved to be able to reproduce shear softening and passive confinement with different confining materials in an accurate manner without recalibration of dilatancy parameters.

The material properties are obtained from the experimental reported data on each case. The rest of the parameters needed to define the constitutive behavior are addressed in Appendix B.

\subsection{Sections under shear or torsion}

\subsubsection{Elastic triangle under pure torsion}

An equilateral triangular elastic section is tested under pure torsion, whose closed-form analytical solution was obtained in [33]. The section height is $200 \mathrm{~mm}$, four points are selected as interpolation grid to construct the bsplines leading to 83 degrees of freedom, and 219 isotropic elastic fibers with the material properties described in Table 1 are used. See Fig.(2).

Table 1: Material properties validation case 4.1.1

\begin{tabular}{|c|c|c|}
\hline $\mathrm{E}[\mathrm{MPa}]$ & $\mathrm{G}[\mathrm{MPa}]$ & $\nu$ \\
\hline 30000 & 12500 & 0.2 \\
\hline
\end{tabular}

A torsional curvature of $\phi_{x}=1 e^{-6} \mathrm{rad} / \mathrm{mm}$ is applied on the centroid of the section. It can be seen, in Fig.(3), that by means of the warpingdistortion displacement field, the tangential stresses and the bi-dimensional shear stress flow are obtained and agree with the theoretical stress pattern.

The maximum tangential stresses as well as the torsional moment are obtained with the presented model and with the model FE TINSA [21]. Besides, four additional b-spline models are analyzed varying first the number 


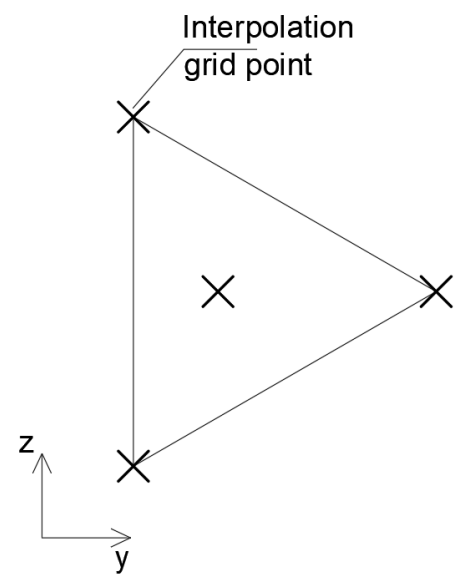

(a)

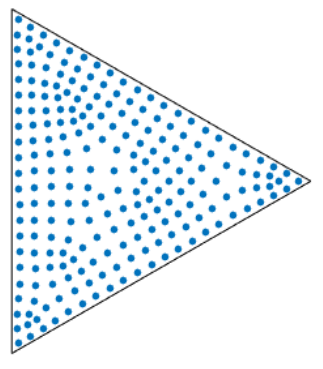

(b)

Figure 2: Triangular section: a) Interpolation grid, b) Fiber distribution.

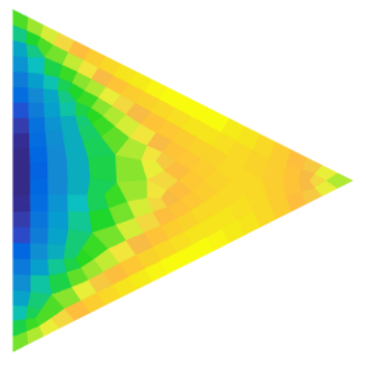

(a) a)

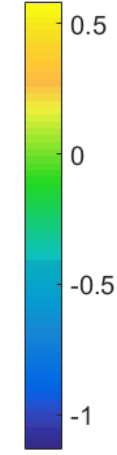

.5

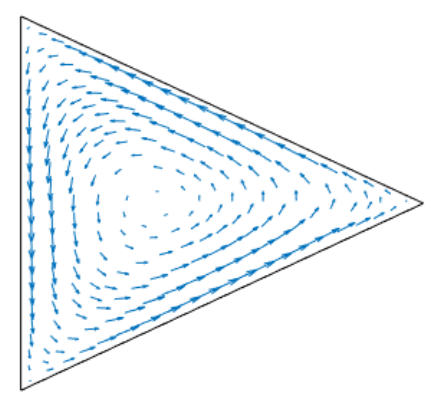

(b)

Figure 3: Triangle under pure torsion: a) $\tau_{x z}$ stresses [MPA], b) Shear stress flow.

of fibers and then the number of degrees of freedom involved in the solution. Table 2 presents numerical results compared against the theoretical solution obtained by [33]. The computational time of each numerical model is addressed as well.

A good agreement with the theoretical solution is obtained regarding the predicted torsional moment. Concerning the maximum stress value all the numerical solutions predict a smaller value than the closed-form solution. This difference can be explained as the maximum value on the theoretical solution is obtained exactly on the edge of the section, while the numerical 
Table 2: Triangular Section in Torsion

\begin{tabular}{|r|c|c|c|c|c|c|c|}
\hline & Analytical $^{\mathrm{a}}$ & FE TINSA & B-Splines & B-Splines & B-Splines & B-Splines & B-Splines \\
\hline DOF & & 767 & 83 & 83 & 83 & 56 & 134 \\
\hline Fibers & & 219 & 219 & 1007 & 91 & 219 & 219 \\
\hline$T_{x}[\mathrm{KNm}]$ & 0.770 & 0.764 & 0.759 & 0.767 & 0.731 & 0.758 & 0.764 \\
\hline Error [\%] & & 0.78 & 1.43 & 0.39 & 5.06 & 1.56 & 0.78 \\
\hline$\tau_{x z}^{\text {max }}[\mathrm{MPa}]$ & 1.250 & 1.128 & 1.129 & 1.192 & 1.044 & 1.131 & 1.153 \\
\hline Error ${ }^{b}[\%]$ & & 9.76 & 9.68 & 4.64 & 16.5 & 9.52 & 7.76 \\
\hline Error ${ }^{c}[\%]$ & & 0.18 & 0.27 & 0.04 & 0.32 & 0.05 & 0.49 \\
\hline Time $[\mathrm{sec}]$ & & 10.7 & 0.37 & 1.54 & 0.17 & 0.31 & 1.02 \\
\hline
\end{tabular}

a Theoretical solution by Timoshenko and Goodier [33]

b Error with respect to the maximum theoretical stress value.

${ }^{c}$ Error with respect to the theoretical stress value on the same location of the fiber.

solutions calculate stresses on discrete locations which are inside the crosssection. The stress field presented by [33] varies quadratically with the $y$ coordinate, which explains that small distances from the edge produce significant variations on the stresses. In Table 2 , the error calculated against the theoretical stress value on the exact same location of the fiber shows that the proposed model captures the stress field in a good manner.

In this case, it can be seen that the most effective way to improve the numerical solution is increasing the number of fibers. This is due to the fact that the displacement field on the theoretical solution [33] has a cubic shape, which can be represented with a reduced number of b-spline functions.

A significant reduction in the number of internal degrees of freedom used can be seen in comparison with the FE solution using the TINSA model. However, results remain on the same level of accuracy. The reduction is translated to the computational time, the b-splines model is faster than the FE model in identical computational conditions.

\subsubsection{Reinforced concrete section in pure shear}

In the following, the response of a reinforced concrete section tested by Kani [34] under pure shear loading, is simulated with the proposed model. The analyzed cross-section is situated on the inflection point of a beam in order to reproduce the pure shear load. The geometry and reinforcement arrangement can be seen in Fig.(4a). In addition, the reported values of the material properties are indicated in Table (3). 
Table 3: Material properties validation case 4.1.2

\begin{tabular}{|l|c|c|}
\hline \multirow{4}{*}{ Concrete } & $f_{c}$ & $28.2 \mathrm{MPa}$ \\
\cline { 2 - 3 } & $f_{t}$ & $1.75 \mathrm{MPa}$ \\
\cline { 2 - 3 } & $E_{c}$ & $25600 \mathrm{MPa}$ \\
\cline { 2 - 3 } & $\nu$ & 0.2 \\
\hline \multirow{2}{*}{ Longitudinal Steel } & $f_{y}$ & $442 \mathrm{MPa}$ \\
\cline { 2 - 3 } & $E_{s}$ & $200000 \mathrm{MPa}$ \\
\hline \multirow{2}{*}{ Transverse Steel } & $f_{y}$ & $400 \mathrm{MPa}$ \\
\cline { 2 - 3 } & $E_{s}$ & $200000 \mathrm{MPa}$ \\
\hline
\end{tabular}

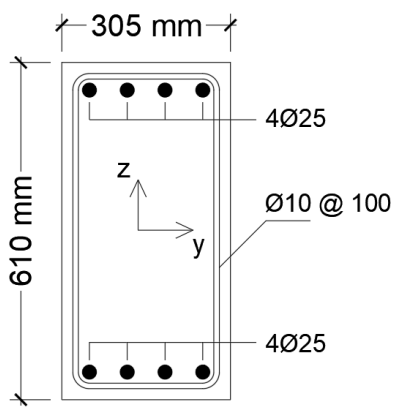

(a)

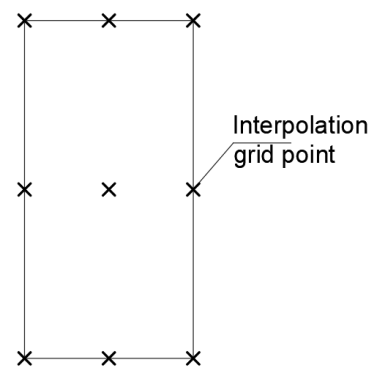

(b)

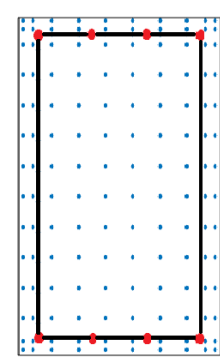

(c)

Figure 4: Specimen: a) Cross-section, b) Interpolation grid, c) Fiber distribution.

The interpolation grid consist on 9 points, 180 material points were used consisting on 140 concrete fibers, 32 fibers for the stirrups and 8 for the longitudinal reinforcement, see Fig.(4b) and (4c). The simulation is performed applying shear strains to the section instead of shear loads in order to capture eventual softening. A total of 70 steps are used to apply the shear strains.

The numerical shear force-strain curve obtained with the presented model is compared against the experimental response in Fig.(5). Further, the numerical response obtained with the model FE TINSA [20] is plotted. Good prediction of the ultimate load was obtained with the presented model. Small differences between both numerical responses exists even though the number of degrees of freedom is appreciably less in the proposed model (83 DOF) than in the FE TINSA model with the same number of fibers (503 DOF). The computational time is reduced nearly 3 times using the B-spline model compared to the FE solution in similar computational conditions. It is worth to mention that the computational time depends not only on the number of 
DOF but also on the number of material points or fibers.

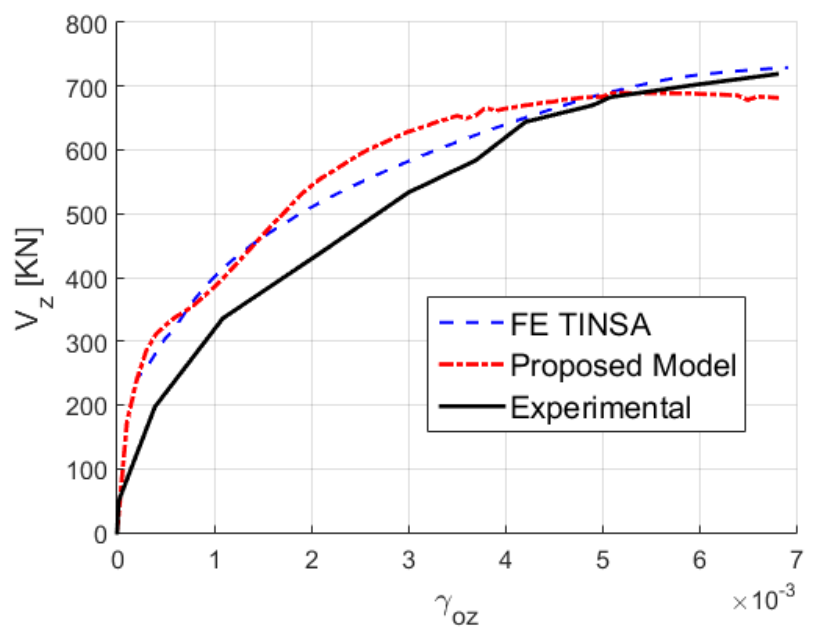

Figure 5: Shear force-strain curve. Experimental results by [34]

Fig.(6) shows part of the information obtained with the presented model. In particular, Fig.(6a) presents the stress distribution on the stirrups at a load level where the vertical branches of the stirrups begin to yield. On Fig.(6b) the tangential stress $\tau_{x z}$ pattern is shown. It can be seen that it varies along the vertical axis and on the horizontal one, stresses concentrate where the theoretical compressive strut intersects the section.

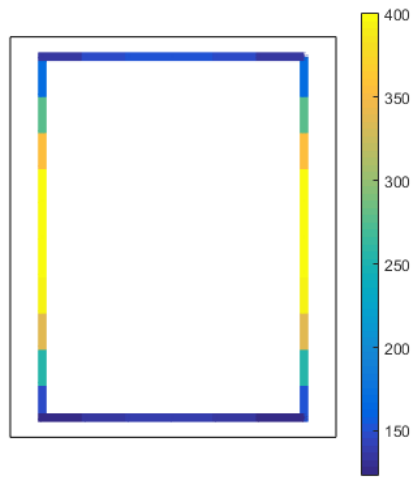

(a) $\sigma_{s t}[\mathrm{MPa}]$

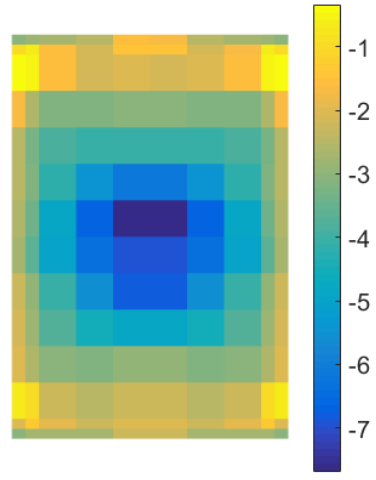

(b) $\tau_{x z}[\mathrm{MPa}]$

Figure 6: a) Stirrups stresses, b) Concrete tangential stresses 


\subsubsection{Reinforced concrete section under combined torsion and bending mo- ments}

The response of a reinforced concrete section under the combined action of torsion and bending moments tested by Onsongo [15] is simulated using the proposed approach. The selected specimen, named TBU2, was part of a larger experimental campaign where different bending to torsion ratios were studied. The main features of the studied cross-section can be seen in Fig.(7a), the material properties are summarized in Table(4).

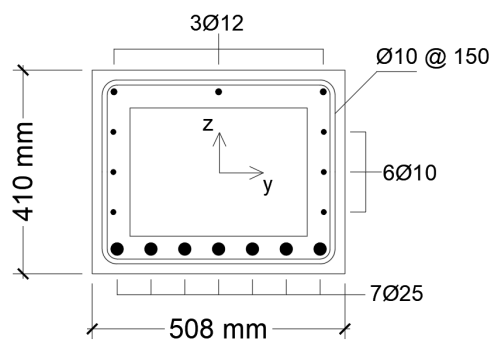

(a)

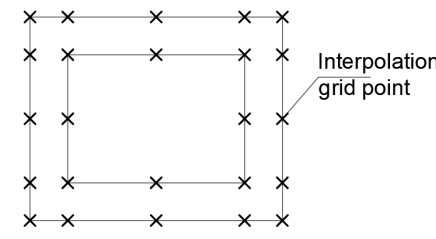

(b)

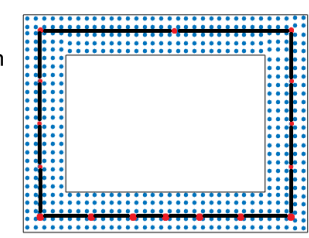

(c)

Figure 7: Specimen: a) Cross-section, b) Interpolation grid, c) Fiber distribution.

Table 4: Material properties validation case 4.1.3

\begin{tabular}{|l|c|c|}
\hline Concrete & $f_{c}$ & $34.8 \mathrm{MPa}$ \\
\hline \multirow{4}{*}{ Longitudinal Steel } & $\varnothing 25 f_{y}$ & $436 \mathrm{MPa}$ \\
\cline { 2 - 3 } & $\varnothing 12 f_{y}$ & $393 \mathrm{MPa}$ \\
\cline { 2 - 3 } & $\varnothing 10 f_{y}$ & $552 \mathrm{MPa}$ \\
\cline { 2 - 3 } & $E_{s}$ & $200000 \mathrm{MPa}$ \\
\hline \multirow{2}{*}{ Transverse Steel } & $\varnothing 10 f_{y}$ & $379 \mathrm{MPa}$ \\
\cline { 2 - 3 } & $E_{s}$ & $200000 \mathrm{MPa}$ \\
\hline
\end{tabular}

The interpolation grid can be seen in Fig.(7b). The fibers distribution is shown in Fig.(7c); it consists of 500 concrete fibers, 104 stirrup fibers and 16 fibers representing the longitudinal reinforcement.

The analysis of the section is performed controlling the torsional curvature and constraining the internal force vector to represent the bending to torsion ratio of the selected specimen which was $R=T / M=0.261$. A total of 220 increments are applied to the section before it reaches the ultimate curvature. 


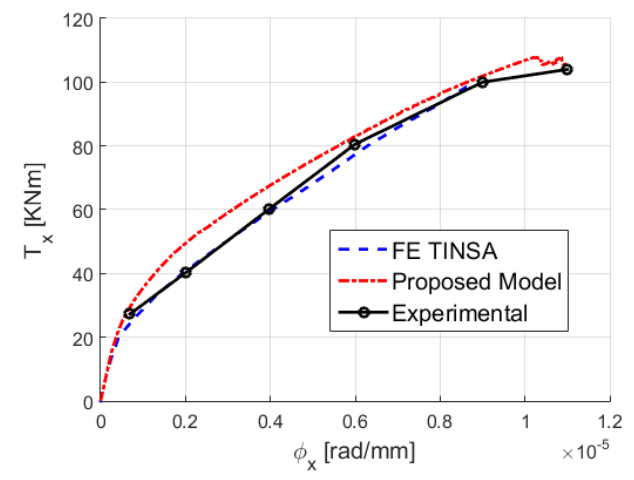

(a)

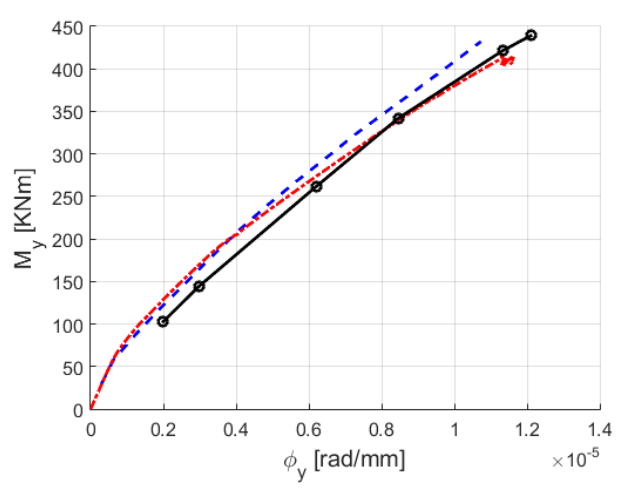

(b)

Figure 8: a)Torsion-curvature and b) Moment-curvature curves. Experimental results by $[15]$

Fig.(8) shows the response curves obtained with the proposed model, the experimental curves and the obtained with the TINSA model by [22] are traced as well, with good agreement with the experimental results. Good predictions of both the ultimate load and curvature are obtained. The response predicted by the proposed model shows numerical oscillations near the ultimate load. These are due to the failure of some material points. On the contrary, the TINSA model fails to converge once the ultimate load is reached. The convergence test in the TINSA model is performed evaluating the unbalanced nodal forces of the finite element mesh. The proposed model performs the convergence test using energetically conjugated forces to the weight factors of the b-splines in Eq.(13), which are obtained integrating over the whole cross-section. This makes the proposed model less sensitive to the failure of a single material point than the TINSA model.

As in the previous cases, a significant decrease of the degrees of freedom used by the proposed model (243 DOF) with respect to the FE model of TINSA [22] with the same number of fibers (1808 DOF) is made. A speedup of around 5 is obtained with the proposed model in comparison with the FE model in similar computational conditions.

The section state at the ultimate load is shown in Fig.(9). Figs.(9a) and (9b) shows the shear stresses, it can be seen that the upper flange carries slightly bigger stresses than the bottom flange, this is due to the bending moment that produces compression on the upper flange, see Fig.(9c), and 


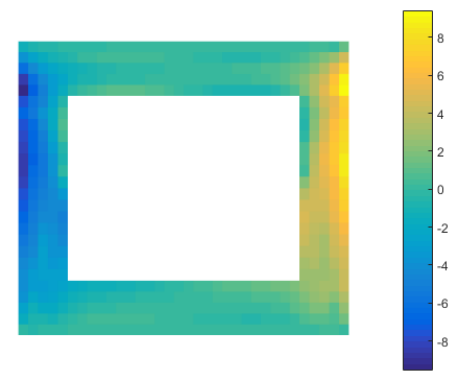

(a) $\tau_{x z}[\mathrm{MPa}]$

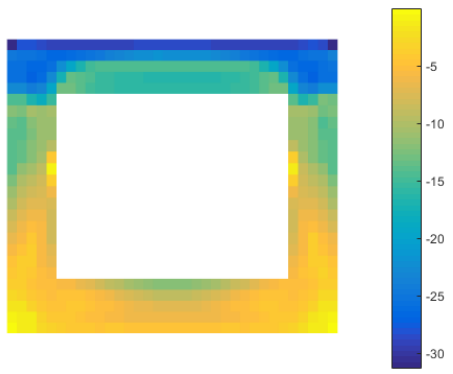

(c) $\sigma_{x x}[\mathrm{MPa}]$

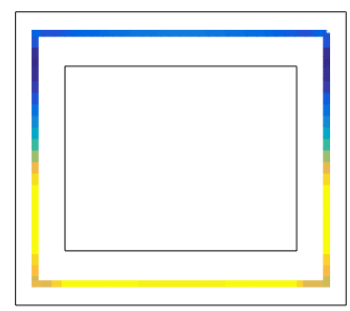

(e) $\sigma_{s t}[\mathrm{MPa}]$

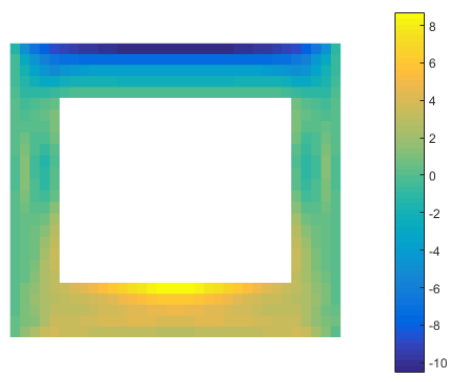

(b) $\tau_{x y}[\mathrm{MPa}]$

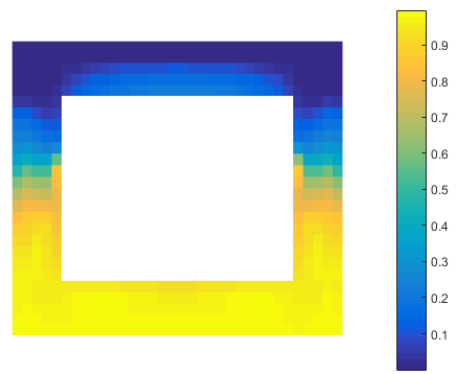

(d) Concrete Damage

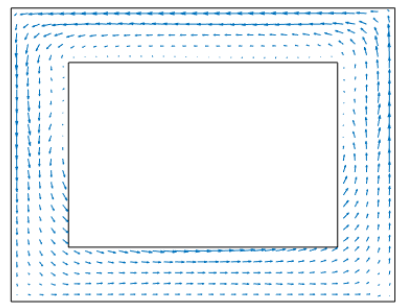

(f) Shear stress flow

Figure 9: Section state for ultimate load: a) Shear stresses $\tau_{x z}$, b) Shear stresses $\tau_{x y}$, c) Longitudinal stresses $\sigma_{x x}$, d) Concrete tensile damage, e) Stirrups stress, f) Shear stress flow

tension in the lower flange which is also fully damaged as it is shown in Fig.(9d); consequently, the upper flange is less damaged than the bottom one. Besides, the presence of shear stresses in the bottom flange is an evidence of the aggregate interlock phenomena. This is reproduced at the material level by means of the evolutive dilatancy parameter introduced in [32]. The 
contribution of the stirrups, see Fig.(9e), shows that the horizontal lower branch has fully yielded, also the vertical branches have reached the yielding stress but plasticity is not developed in its full length.

\subsection{Study of confinement of concrete sections}

As it was previously stated, the complementary displacement field reproduces the distortion of the section. This enables the model to capture explicitly the contribution of transverse reinforcements. A particular problem where this takes relevance is the case of confinement in concrete sections.

Confinement is of major importance in the design and assessment of new, existing, and repaired reinforced concrete structures, as it increases both strength and ductility. Confinement effects are consequence of the interaction between concrete and transverse reinforcement; when compression is applied to concrete, it has a transverse expansion, which is constrained by the transverse reinforcement providing the confinement pressure that increases the strength and ductility of the material. Transverse reinforcement are usually provided by steel stirrups or spirals in the case of new structures, or by steel or fiber-reinforced-polymers (FRP) jackets typically in the reinforcement of existing structures.

Classic fiber-beam models simulate confinement through 1D constitutive models that include transverse reinforcement by means of reinforcements ratios at the material level. Currently, those constitutive laws have to be calibrated for different confining materials or for different arrangements of the transverse reinforcement $[35 ; 36]$. This approach is not objective, as it masks the 3D behavior of concrete while it interacts with the confinement material. Hence, if different confinement materials are used, the model needs to be recalibrated.

In order to test the capabilities of the presented model to represent confinement in an objective manner, three concrete sections with different confining materials and arrangements are simulated under pure axial loading.

\subsubsection{Confinement of a circular column with steel spiral}

In the following, a circular section of a reinforced concrete column tested by Mander et al. [37] is simulated with the presented model. This specimen is the unit number 4 of a larger experimental campaign which was the basis of the well-known 1D constitutive model for confined concrete of Mander et al. [35]. Table (5) shows the material properties. 
Table 5: Material properties validation case 4.2.1

\begin{tabular}{|l|c|c|}
\hline Concrete & $f_{c}$ & $28 \mathrm{MPa}$ \\
\hline \multirow{2}{*}{ Longitudinal Steel } & $f_{y}$ & $295 \mathrm{MPa}$ \\
\cline { 2 - 3 } & $E_{s}$ & $200000 \mathrm{MPa}$ \\
\hline \multirow{2}{*}{ Transverse Steel } & $f_{y}$ & $320 \mathrm{MPa}$ \\
\cline { 2 - 3 } & $E_{s}$ & $200000 \mathrm{MPa}$ \\
\hline
\end{tabular}

The test setup can be seen in Fig.(10a), the interpolation grid consisting on 5 points is shown in Fig.(10b). The interpolation grid is set to trace the higher strain gradient that it is produced on the perimeter of the section. The fiber distribution in Fig.(10c) consists of 484 concrete fibers, 60 stirrup fibers and 12 fiber representing the longitudinal reinforcement. The test is performed applying axial strains in 45 increments, then the model fails to converge.

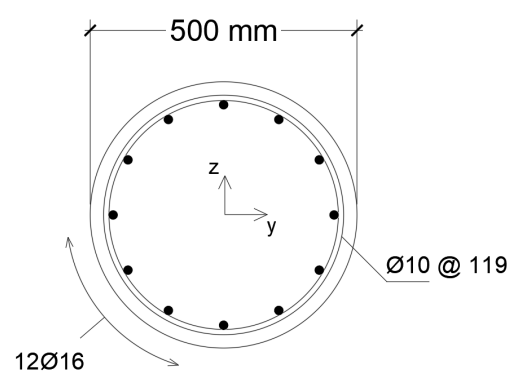

(a)

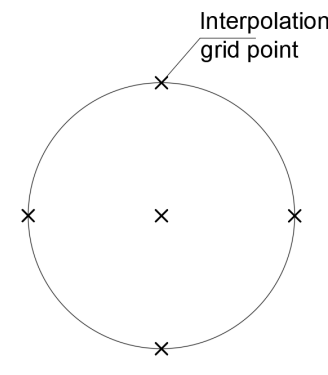

(b)

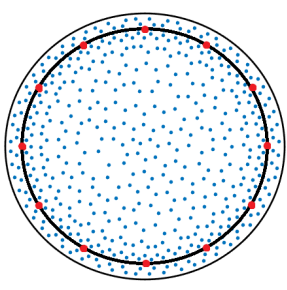

(c)

Figure 10: Specimen: a) Cross-section, b) Interpolation grid, c) Fiber distribution.

Three pairs of numerical and experimental curves are presented in Fig.(11a). The total axial load carried by the whole section is plotted against the axial strain, also the axial load carried by the longitudinal reinforcements and by the concrete cover are plotted as well. Fig.(11b) shows the predicted strains on the transverse reinforcements compared against the experimental curve.

The model shows to predict in a good way the strength and the post-peak stiffness. However, it can be seen that the model fails to converge before the ultimate experimental strain is reached. The total axial load, the load carried by the reinforcements and the strains in the transverse reinforcement have a good agreement with the experimental data. In the case of the concrete cover, 


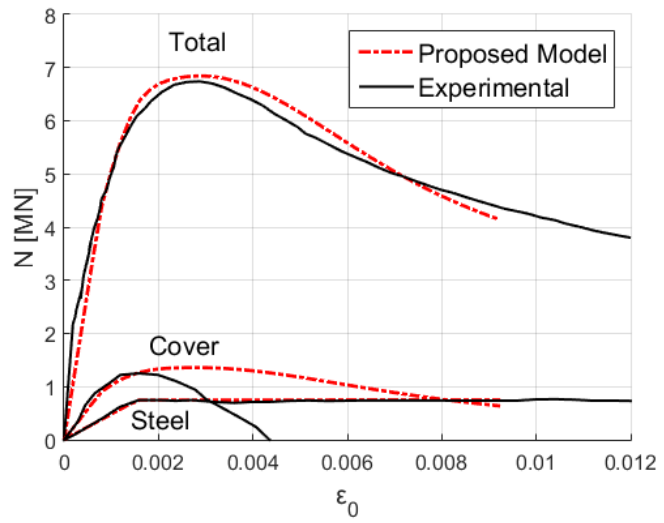

(a)

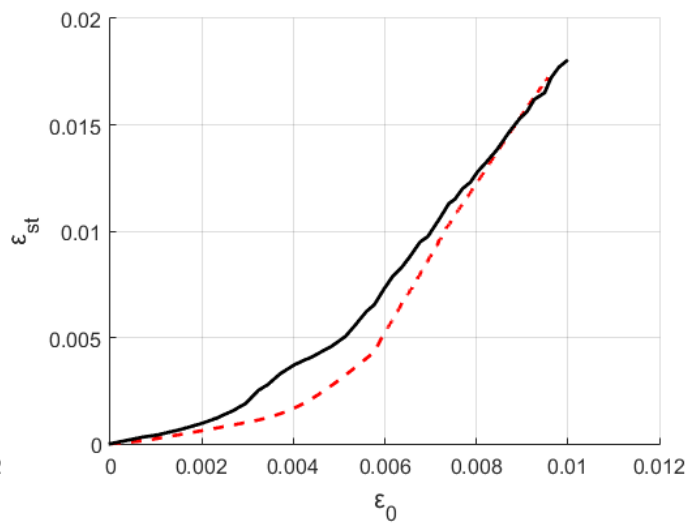

(b)

Figure 11: (a) Axial force-strain curves. (b) Spiral strains. Experimental results by [37]

it can be seen that the maximum strength is well captured but the postpeak behavior predicted by the proposed model differs from experimental evidence. This can be explained by the fact that the proposed model solves the equilibrium equations at the sectional level in a weak form as in Eq.(4), thus the local error in a single fiber could be greater than the error at the sectional level, as it is evidenced in this case.

Fig.(12a) shows the longitudinal stress field and Fig.(12d) shows the longitudinal stress along a radius of the section. It can be seen that longitudinal stresses are higher on the section core than on the perimeter of the section, being minimum in the section cover, this coincides with the observed response. In Fig.(12c) the stresses on the steel spiral are presented at the ultimate state, it can be seen that the spiral has fully yielded. Fig.(12b) shows the radial strains are bigger on the cover than on the core of the section, this is evidence of the existance of more damage in that region or spalling of concrete.

Fig. $(12 \mathrm{e})$ shows the confining stresses in the radial $\left(\sigma_{r r}\right)$ and circumferential $\left(\sigma_{\theta \theta}\right)$ directions at different distances from the center. Theoretically, this curves should be constant, but it can be seen that oscillations appear as the distance from the center increases. The reason of the oscillations is the fact that the interpolation functions in Eq.(15) are constructed in a Cartesian reference system rather than in a cylindrical one, which in this case would be more suitable. Nevertheless, the average $\sigma_{r r}$ at radius of $r / R=0.9$, 


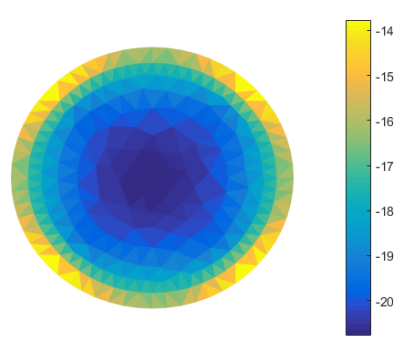

(a) $\sigma_{x x}[\mathrm{MPa}]$

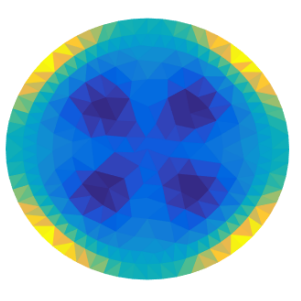

(b) $\varepsilon_{r r}$
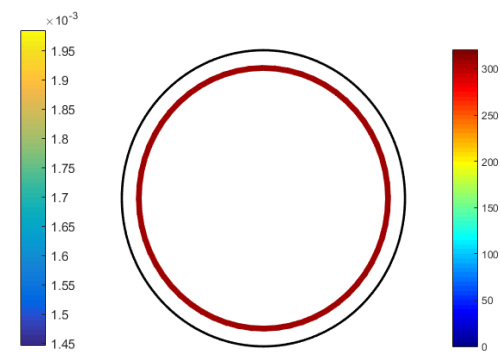

(c) $\sigma_{s t}[\mathrm{MPa}]$

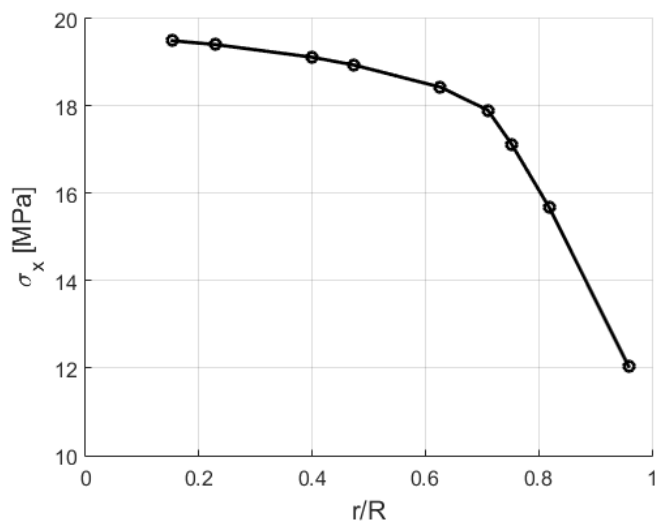

(d)

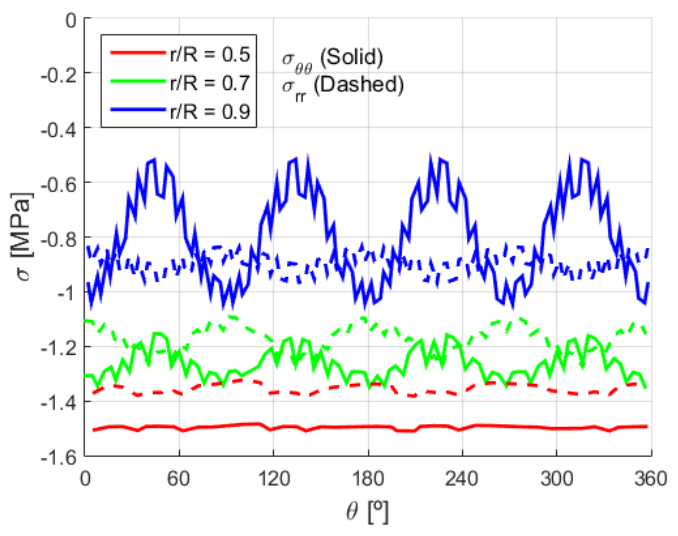

(e)

Figure 12: Section state: a) Longitudinal stresses, b) Radial strains, c) Stirrups stresses, d) Longitudinal stresses-radius curve, e) $\sigma_{r r}$ and $\sigma_{\theta \theta}-\theta$ curves.

which coincides with the position of the transverse reinforcement, is equal to $0.90 M P a$. This almost coincides with the lateral confining stress predicted by [35] which was $0.85 \mathrm{MPa}$.

The proposed model shows to be able to predict well the overall behavior with a single definition of the concrete properties. In contrast, classical fiberbeam models [8] define different zones in the cross-section with different material parameters in order to capture the confinement phenomena.

\subsubsection{Confinement of a circular section with a CFRP jacket}

A circular concrete section wrapped with a CFRP sheet tested by Micelli and Modarelli [38] is simulated with the proposed model. The specimen, named CC1, is part of a larger experimental campaign studying the behaviour of FRP confined concrete. The test setup can be seen in Fig.(13a), and the 
material properties are shown in Table (6). The interpolation points and the fiber distribution can be seen in Figs.(13b) and (13c). The axial strain is applied in 50 increments when the ultimate strain is reached.

Table 6: Material properties validation case 4.2 .2

\begin{tabular}{|l|c|c|}
\hline Concrete & $f_{c}$ & $28 M P a$ \\
\hline \multirow{2}{*}{ CFRP } & $f_{u}$ & $3070 M P a$ \\
\cline { 2 - 3 } & $E$ & $221000 M P a$ \\
\hline
\end{tabular}

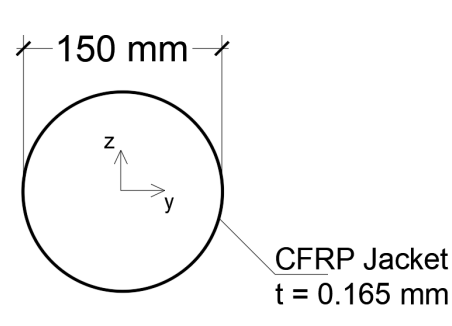

(a)

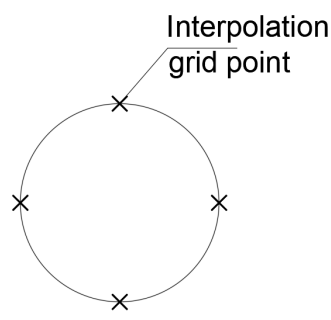

(b)

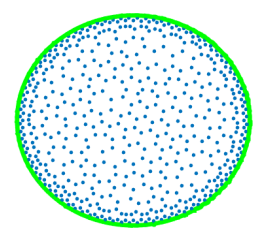

(c)

Figure 13: Specimen: a) Cross-section, b) Interpolation grid, c) Fiber distribution.

The numerical axial force-strain curve is compared against the experimental one in Fig.(14). Good prediction of the ultimate load is obtained. The increase on the ductility is also well captured. The analysis stopped when the transverse jacket reached its ultimate stress and softening starts to develop, as it is evident in Fig.(15b), this coincides with the observed experimental failure. Hence, the model allows assessing the capacity of the element without the need of the empirical effective strain concept in the FRP [39].

Figs.(15a) and (15b) show the behaviour of the section at the ultimate load. The longitudinal stresses are almost constant over the cross-section as the confining element is on the perimeter of the section giving a uniform confinement to the whole section, small variations of the stress are due to the interpolation.

\subsubsection{Confinement of a concrete filled steel tube column}

In the following, a rectangular concrete filled steel tube (CFST) is simulated and compared against the experimental results of the specimen HSS1 


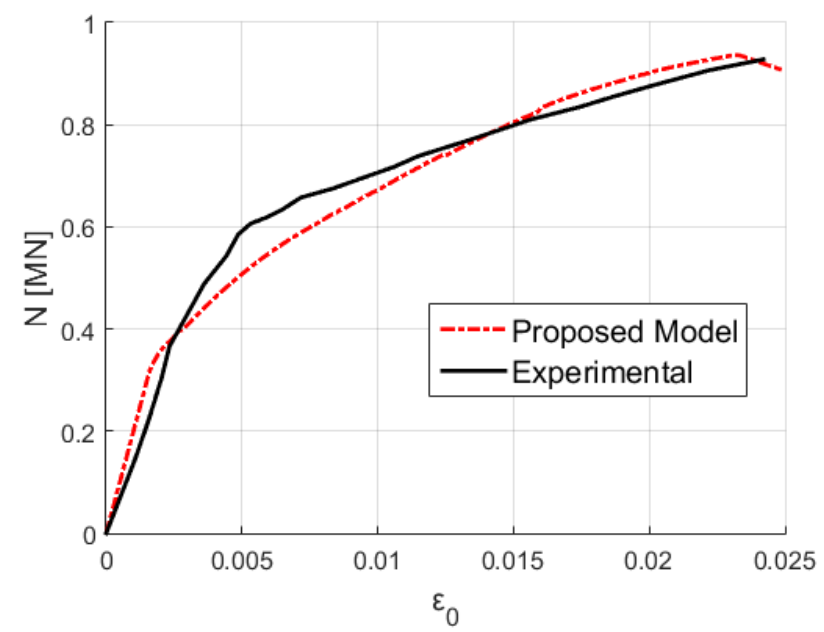

Figure 14: Axial force-strain curve. Experimental results by [38]

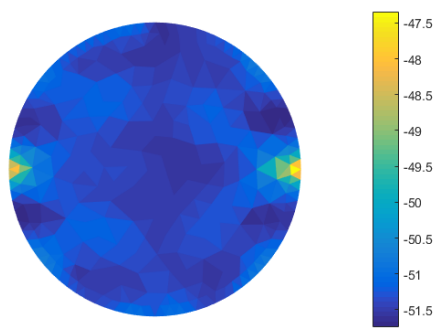

(a) $\sigma_{x x}[\mathrm{MPa}]$

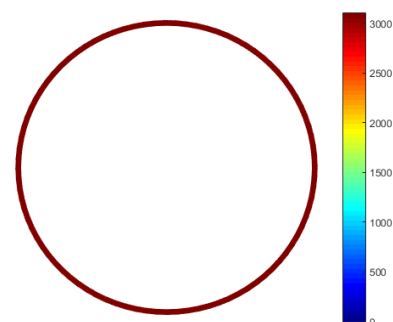

(b) $\sigma_{s t}[\mathrm{MPa}]$

Figure 15: Section state: a) Longitudinal stresses, b)Stirrups stresses.

tested by Uy [40]. The test setup can be seen in Fig.(16a) and the material properties are addressed in Table (7). The analysis is performed applying 45 axial strain increments.

The numerical simulation is performed using the interpolation grid as in Fig.(16b) and the fiber distribution as in Fig.(16c). In the experimental test, both the steel and concrete were submitted to the axial load. To model the steel plates, a classic Von-Mises constitutive law with isotropic hardening is used.

Fig.(17) shows both the numerical and experimental axial force-strain curves. Good agreement is achieved by the sectional model. The enhancement in strength and ductility is well captured. 
Table 7: Material properties validation case 4.2.3

\begin{tabular}{|l|c|c|}
\hline Concrete & $f_{c}$ & $28 \mathrm{MPa}$ \\
\hline \multirow{3}{*}{ Steel jacket } & $\sigma_{y}$ & $750 \mathrm{MPa}$ \\
\cline { 2 - 3 } & $E$ & $200000 \mathrm{MPa}$ \\
\cline { 2 - 3 } & $\nu$ & 0.3 \\
\hline
\end{tabular}

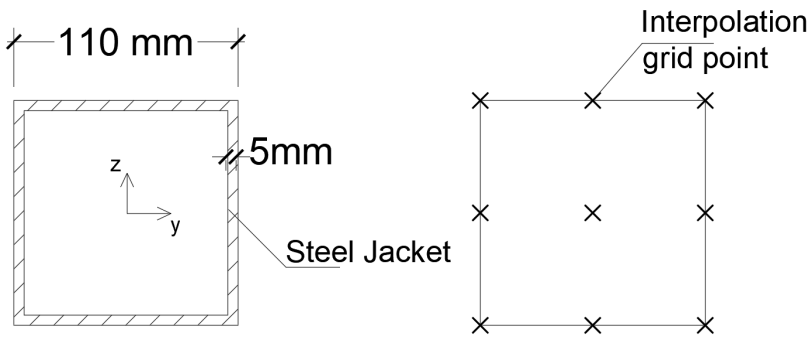

(a)

(b)

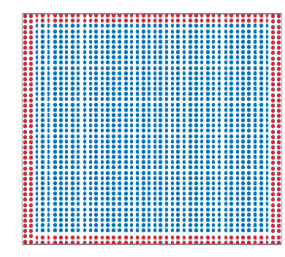

(c)

Figure 16: Specimen: a) Cross-section, b) Interpolation grid, c) Fiber distribution.

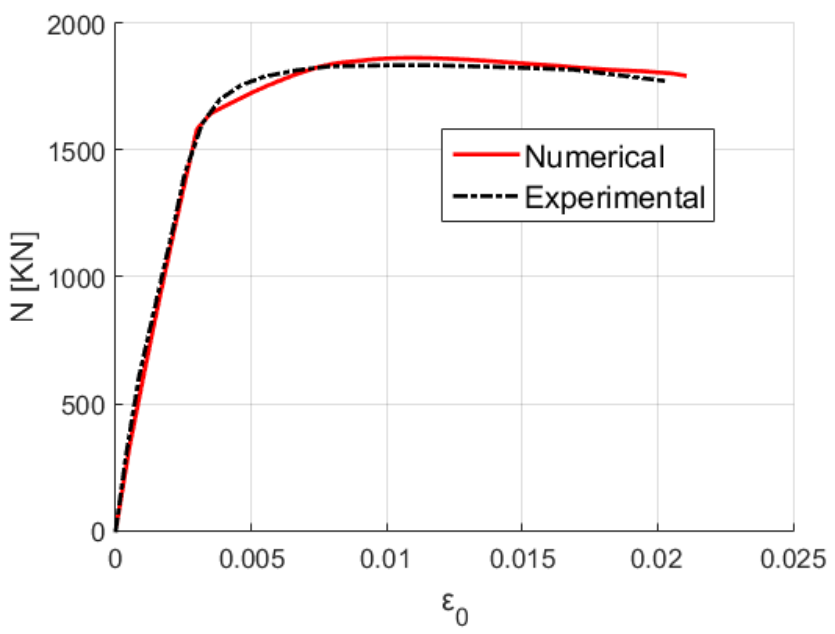

Figure 17: Axial force-strain curve. Experimental results by [40]

The longitudinal stresses at the ultimate state in the steel jacket and in the concrete core can be seen in Fig.(18a). The nonuniform confinement given by the jacket causes regions with different degrees of compressive damage in concrete, this can be seen in Figs.(18b-18c). 


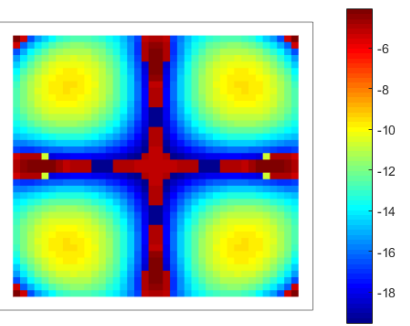

(a) $\sigma_{x x}[\mathrm{MPa}]$

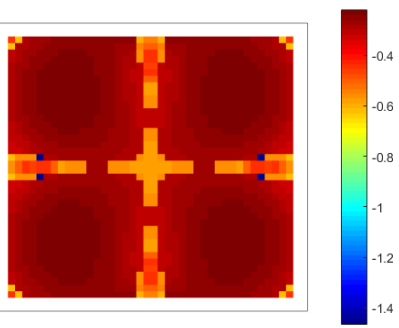

(b) $\sigma_{y y}[\mathrm{MPa}]$

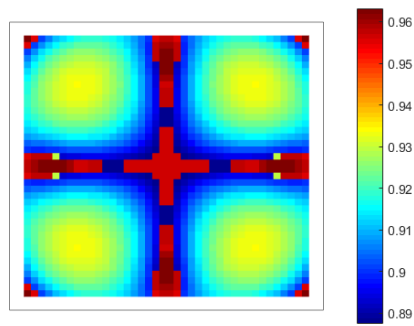

(c) $\kappa_{c}$

Figure 18: Section state: a) Longitudinal stresses, b) Transverse stresses, c) Compression damage in concrete fibers.

\section{Conclusions}

A new sectional model for the nonlinear analysis of reinforced concrete elements is proposed. The model is based on the original model of Bairán and Marí [20, 21, 22], which proposed a formulation based on the decomposition of the displacement field of a beam into a plane-section displacement field and a complementary warping-distortion field. The complementary field is obtained by considering explicitly the inter-fiber equilibrium which in the original model was solved by means of a $2 \mathrm{D} \mathrm{FE}$ model. In order to make a more efficient sectional formulation, an alternative solution is proposed in this paper.

The displacement field is here calculated as a weighted sum of b-splines functions defined on the cross-section domain, this method avoids the FE solution reducing significantly the number of unknowns involved in the sectional problem.

The model considers a displacement field independent of the beam-axis coordinate, which allows the formulation of a completely local model, which can be used as a cross-section constitutive model on each integration point of any standard frame formulation without the need of additional degrees of freedom at the frame level.

The model is validated through a series of experiments where the capabilities of the model are tested and compared against experimental results. It is shown that the model is able to trace the response of reinforced concrete section under complex loading until failure. Pure shear loading and coupling between torsion and bending is accurately reproduced.

A remarkable reduction in the DOFs involved in the solution compared 
with the FE model is obtained. This enabled the model to have significant speedups proving its lower computational demand with respect to the FE solution.

The sectional model is used to study confinement in reinforced concrete sections. As the formulation considers the distortion of the section, it is able to capture the interaction of transverse reinforcements and the concrete mass. Then confinement is simulated in an objective manner for different confining materials, section shape and reinforcement arrangements.

The sectional model presented in this work constitutes and efficient framework capable of reproducing the nonlinear behaviour of concrete elements under different loading states and reproducing complex failure modes. This in conjunction with its reduced computational demand, in comparison with other solution methods, makes it suitable for the analysis of complete structures.

\section{Acknowledgments}

The authors acknowledge the support provided by Spanish Ministry of Economy, Industry and Competitiveness and the European Regional Development Fund (ERDF), through the projects BIA2015-64672-C4-1R and BIA2012-36848. The first author acknowledges the support of the Catalan Agency for University and Research through doctoral scholarship 2018FI-B100103.

\section{Declarations of interest}

Declarations of interest: None

\section{References}

[1] I. Carol, J. Murcia, Nonlinear time-dependent analysis of planar frames using an 'exact' formulation - i. theory, Computers \& Structures 33 (1989) $79-87$.

[2] A. R. Marí, Nonlinear geometric, material, and time dependent analysis of three dimensional reinforced and prestressed concrete frames, Technical Report SESM 84-12, University of California, Berkeley, 1984. 
[3] E. Spacone, V. Ciampi, F. Filippou, Mixed formulation of nonlinear beam finite element, Computers \& Structures 58 (1996) $71-83$.

[4] EN 1992-1-1 Eurocode 2: Design of concrete structures - Part 1-1: General ruels and rules for buildings, EN, CEN, Brussels, 2005.

[5] CEB-FIB, Model Code 2010, Thomas Telford, London, 2010.

[6] ACI Committee 318, Building code requirements for structural concrete : (ACI 318-14) ; and commentary (ACI 318R-14), American Concrete Institute, Farmington Hills, 2014.

[7] F. Taucer, E. Spacone, F. Filippou, A fiber beam-column element for seismic response analysis of reinforced concrete structures, Technical Report 17, Earthquake Engineering Research Center, 1991.

[8] E. Spacone, F. C. Filippou, F. F. Taucer, Fibre beam column model for non-linear analysis of R/C frames: Part I. formulation, Earthquake Engineering \& Structural Dynamics 25 (1996) 711-725.

[9] Y.-J. Kang, A. C. Scordelis, Nonlinear analysis of prestressed concrete frames, Journal of the structural division 106 (1980).

[10] G. Ranzo, M. Petrangeli, A fibre finite beam element with section shear modelling for seismic analysis of RC structures, Journal of Earthquake Engineering 2 (1998) 443-473.

[11] P. Ceresa, L. Petrini, R. Pinho, Flexure-shear fiber beam-column elements for modeling frame structures under seismic loading — state of the art, Journal of Earthquake Engineering 11 (2007) 46-88.

[12] J. M. Bairán, A. R. Marí, Shear-bending-torsion interaction in structural concrete members: A nonlinear coupled sectional approach, Archives of Computational Methods in Engineering 14 (2007) 249-278.

[13] G. Balduzzi, S. Morganti, J. Füssl, M. Aminbaghai, A. Reali, F. Auricchio, Modeling the non-trivial behavior of anisotropic beams: A simple timoshenko beam with enhanced stress recovery and constitutive relations, Composite Structures 229 (2019) 111265. 
[14] A. T. Karttunen, R. von Hertzen, On the foundations of anisotropic interior beam theories, Composites Part B: Engineering 87 (2016) 299 -310 .

[15] W. Onsongo, The diagonal compression field theory for reinforced concrete beams subject to combined torsion, flexure and axial load, Ph.D. thesis, University of Toronto, Toronto, 1978.

[16] T. T. C. Hsu, Torsion of reinforced concrete, Van No.Strand Reinhold, New York, 1984.

[17] R. Park, T. Paulay, Reinforced Concrete Structures, John Wiley \& Sons, New York, 1975.

[18] F. Vecchio, M. Collins, Predicting the response of reinforced concrete beams subjected to shear using the modified compression field theory, ACI Structural J. 85 (1988) $258-268$.

[19] E. Bentz, Sectional analysis of reinforced concrete members, Ph.D. thesis, University of Toronto, Toronto, 2000.

[20] J. M. Bairán, A. R. Marí, Coupled model for the non-linear analysis of anisotropic sections subjected to general 3D loading. Part 1: Theoretical formulation, Computers \& Structures 84 (2006) $2254-2263$.

[21] J. M. Bairán, A. R. Marí, Coupled model for the nonlinear analysis of sections made of anisotropic materials, subjected to general 3D loading. Part 2: Implementation and validation, Computers \& Structures 84 (2006) $2264-2276$.

[22] J. M. Bairán, A. R. Marí, Multiaxial-coupled analysis of RC crosssections subjected to combined forces, Engineering Structures 29 (2007) $1722-1738$.

[23] S. Mohr, J. M. Bairán, A. R. Marí, A frame element model for the analysis of reinforced concrete structures under shear and bending, Engineering Structures 32 (2010) 3936 - 3954.

[24] V. Le Corvec, Nonlinear 3D frame element with multi-axial coupling under consideration of local effects, Ph.D. thesis, Department Civil and Environmental Engineering, University of California, Berkeley, 2012. 
[25] P. Di Re, 3D beam-column finite elements under tri-axial stress-strain states: non-uniform shear stress distribution and warping., Ph.D. thesis, Department of Structural and Geotechnical Engineering, Università la Sapienza, Roma, 2017.

[26] A. Kagermanov, P. Ceresa, 3D fiber-based frame element with multiaxial stress interaction for RC structures, Advances in Civil Engineering 2018 (2018).

[27] G. E. Farin, NURBS: From Projective Geometry to Practical Use, A. K. Peters, Ltd., Natick, MA, USA, 2nd edition, 1999.

[28] X. Wei, Y. Zhang, L. Liu, T. J. Hughes, Truncated T-splines: Fundamentals and methods, Computer Methods in Applied Mechanics and Engineering 316 (2017) 349 - 372. Special Issue on Isogeometric Analysis: Progress and Challenges.

[29] T. J. Hughes, J. A. Evans, A. Reali, Finite element and NURBS approximations of eigenvalue, boundary-value, and initial-value problems, Computer Methods in Applied Mechanics and Engineering 272 (2014) $290-320$.

[30] K. Höllig, J. Hörner, Programming finite element methods with weighted B-splines, Computers \& Mathematics with Applications 70 (2015) 1441 - 1456. High-Order Finite Element and Isogeometric Methods.

[31] C. De Boor, A practical guide to splines; rev. ed., Applied mathematical sciences, Springer, Berlin, 2001.

[32] M. Poliotti, J.-M. Bairán, A new concrete plastic-damage model with an evolutive dilatancy parameter, Engineering Structures 189 (2019) $541-549$.

[33] S. Timoshenko, J. Goodier, Teoría de la elasticidad (Theory of elasticity), Ed. Urmo, Bilbao, 1972.

[34] M. Kani, An experimental investigation of reinforced and prestressed beams in shear, Ph.D. thesis, University of Toronto,Toronto, 1977.

[35] J. B. Mander, M. J. N. Priestley, R. Park, Theoretical stress-strain model for confined concrete, Journal of Structural Engineering 114 (1988) 1804-1826. 
[36] L. Bisby, A. Dent, M. Green, Comparison of confinement models for fibre-reinforced polymer-wrapped concrete, ACI Structural Journal 102 (2005) 62-72.

[37] J. B. Mander, M. J. N. Priestley, R. Park, Observed stress-strain behavior of confined concrete, Journal of Structural Engineering 114 (1988) $1827-1849$.

[38] F. Micelli, R. Modarelli, Experimental and analytical study on properties affecting the behaviour of FRP-confined concrete, Composites Part B: Engineering 45 (2013) $1420-1431$.

[39] Y.-F. Wu, J.-F. Jiang, Effective strain of FRP for confined circular concrete columns, Composite Structures 95 (2013) 479 - 491.

[40] B. Uy, Strength of short concrete filled high strength steel box columns, Journal of Constructional Steel Research 57 (2001) 113 - 134.

\section{Appendix A. Sectional Algorithm}

The structure of the algorithm that computes the sectional state is shown in Fig.(A.19). The value of the force tolerance used in all numerical cases is equal (in Newtons) to $t o l=0.001 A_{c}$, where $A_{c}$ is the area of the cross-section.

\section{Appendix B. Material Parameters}

The concrete constitutive law presented in [32] requires the following material parameters, see Eq.(B.1). 


$$
\begin{array}{rlr}
E & =\left(0.8+0.2 \frac{f_{c}+8}{88}\right) \sqrt[3]{f_{c}+8} & {[\mathrm{MPa}]} \\
f_{t} & =0.302 f_{c}^{2 / 3} & {[\mathrm{MPa}]} \\
G_{t} & =0.073 f_{c}^{0.18} & {[\mathrm{~N} / \mathrm{mm}]} \\
G_{c} & =\left(\frac{f_{c}}{f_{t}}\right)^{2} G_{t} & \\
\nu & =0.2 & \\
\bar{D}_{c} & =0.423\left(1-\frac{f_{c}}{\varepsilon_{0} E}\right) & \\
\bar{D}_{t} & =0.5 & \\
\phi^{\text {max }} & =65^{\circ} & \\
\psi^{\text {max }} & =32^{\circ} &
\end{array}
$$

Where $E$ is the elastic modulus; $f_{c}$ is the uniaxial compressive strength; $f_{t}$ is the tensile uniaxial strength; $G_{t}$ is the fracture energy and $G_{c}$ is the compressive counterpart; $\nu$ is the Poisson's coefficient; $\bar{D}_{c}$ and $\bar{D}_{t}$ are the stiffness degradation variables in tension and compression uniaxial cases respectively; $\varepsilon_{0}$ is the axial strain corresponding to the peak compressive strength in uniaxial conditions; $\phi^{\max }$ and $\psi^{\max }$ are the maximum internal friction and dilatancy angles respectively.

In the case of steel, the only parameters needed to the define the material behavior are the elastic modulus $E$, the yielding stress $f_{y}$ and the hardening ratio, which in all cases is equal to $b=0.001$. 


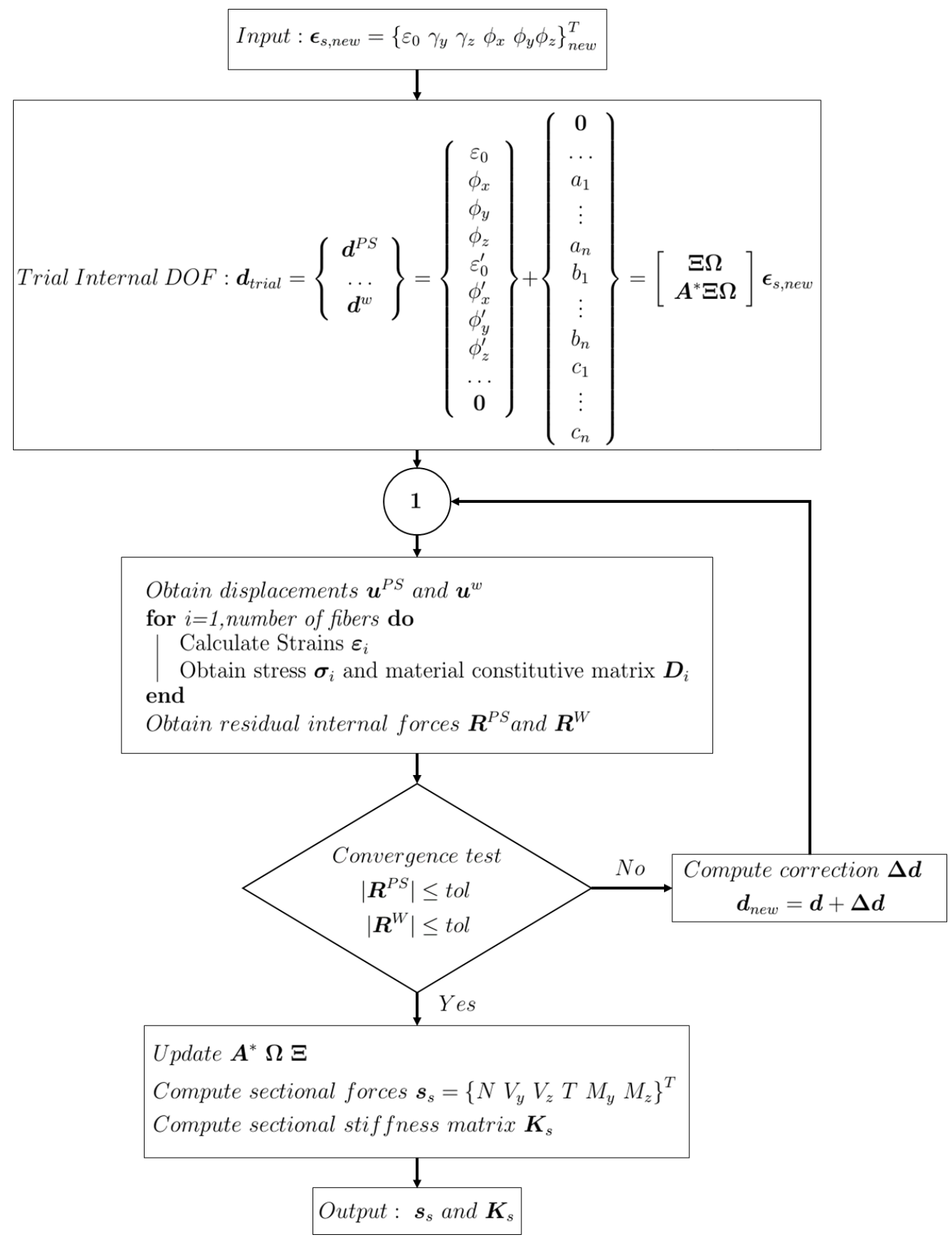

Figure A.19: Sectional model flow chart 